\title{
Towards more uniform deformation in metallic glasses: The role of Poisson's ratio
}

\author{
Yujie Wei ${ }^{\mathrm{a}, *}$, Xianqi Lei ${ }^{\mathrm{a}}$, Li-Shan Huo ${ }^{\mathrm{b}}$, Wei-Hua Wang ${ }^{\mathrm{b}}$, A.L. Greer ${ }^{\mathrm{c}}$ \\ a LNM, Institute of Mechanics, Chinese Academy of Sciences, Beijing 100190, PR China \\ ${ }^{\mathrm{b}}$ Institute of Physics, Chinese Academy of Sciences, Beijing 100080, PR China \\ ${ }^{\mathrm{c}}$ Department of Materials Science $\mathcal{E}$ Metallurgy, University of Cambridge, Pembroke Street, Cambridge CB2 3QZ, U.K
}

\section{A R T I C L E I N F O}

\section{Article history:}

Received 23 July 2012

Received in revised form

25 September 2012

Accepted 26 September 2012

Available online 2 October 2012

Keywords:

Bending test

Metallic glasses

Micromechanical modelling

Plastic deformation

Shear bands

\begin{abstract}
A B S T R A C T
We develop a quantitative analysis of how the plastic deformation in a metallic glass is more uniform if its Poisson ratio $v$ is higher. The plasticity of metallic glasses under ambient conditions is mediated by shear localized in thin bands, and can be characterized by experiments on the bending of thin plates. We extend the analysis by Conner et al. (Conner et al., J. Appl. Phys. 94 (2003), 904-911) of bands in bent plates to include the micromechanics of individual shear bands. Expressions are derived for the shearband spacing and the offset on each band. Both these quantities are predicted to decrease as $v$ is increased. The predictions are tested against measurements on metallic glasses with a wide range of $v$. Good agreement is found, supporting the new model for the shear-band spacing, and pointing the way towards more diffuse deformation, and consequently improved plasticity and toughness, of metallic glasses as $v$ increases toward the limiting value of 0.5 .
\end{abstract}

Crown Copyright $\odot 2012$ Published by Elsevier B.V. All rights reserved.

\section{Introduction}

In metallic glasses (MGs), many atomic-level shear transformation zones are activated under stress, and the initial deformation is uniform. Soon, however, the deformation localizes into rather few macroscopic shear bands (SBs) that lead to catastrophic failure [1-4]. This shear localization is a key factor limiting the wider application of MGs, which otherwise can have attractive mechanical properties. There is much active research on making the plastic deformation more uniform by increasing the population density of SBs. The bending of thin plates is particularly useful for studies of SBs and their spacing, since the propagation of SBs stops as they approach the neutral plane and early catastrophic failure is avoided, permitting substantial deformability when the samples are sufficiently thin [5-8]. Bending experiments are also directly relevant for such applications as MG foams [9,10] and coatings [11].

Noting that resistance to plastic shear is proportional to the shear modulus $\mu$, and that resistance to dilatation and cracking is proportional to the bulk modulus $B$, Pugh [12] in surveying polycrystalline metals suggested that the ratio $\mu / B$ should correlate with the degree of plasticity or brittleness. A low value of $\mu / B$, equally expressed as a high value of Poisson's ratio $v$, favours

\footnotetext{
* Correspondence to: LNM, Institute of Mechanics, Chinese Academy of Sciences, State Key Lab of Nonlinear Mechanics, Bei Si Huan Xi Road \#15, Beijing 100190, China. Tel: +86108254 4169, fax: +861082543977.

E-mail address: yujie_wei@lnm.imech.ac.cn (Y. Wei).
}

plasticity. The link between $v$ and plasticity was noted for metallic glasses by Chen et al. [13], and was explored quantitatively by Lewandowski et al. [14], who found a sharp transition: Metallic glasses show significant toughness only when $v$ exceeds a critical value of $0.31-0.32$. This has excited interest in tuning compositions to increase $v$, and in this way many tough bulk MGs have been realized [15-17].

While the critical value of $v$ separating plastic and brittle behaviour is important, it is equally of interest to consider the extent of plasticity. Indeed, in Pugh's survey [12], none of the polycrystalline metals failed by truly brittle fracture, and the point of interest was the correlation of $v$ with the extent of the plastic range. In the present work we extend considerations of this type to metallic glasses. We explore their plasticity when $v$ exceeds the critical value noted above. The study by Lewandowski et al. [14] suggests that away from the plastic-brittle transition, the toughness continues to rise as $v$ rises. Demetriou et al. [17] have shown that a metallic glass with a particularly high $v$ is tougher than any other. But quantification of the rôle of $v$ has so far been lacking.

For polycrystalline metals, quantitative analyses [18,19] following Pugh [12] consider the stress state at a crack tip and the local conditions for spontaneous emission of dislocations. In metallic glasses, in contrast, plasticity is mediated by the shear bands. Their spacing is of particular interest and, as seen in the work of Demetriou et al. [17], is very fine (i.e. the deformation is more uniform) when $v$ is high. The degree of plasticity of metallic 
glasses is dependent on sample size [20-22], and many aspects of this were analysed in the important study by Conner et al. [5], who derived expressions for shear-band spacing $\lambda$ and shear offset $\Delta u$ in bent plates. They found that both $\lambda$ and $\Delta u$ depend, among other factors, on $v$. While adopting the essential elements of the analysis by Conner et al. [5], we now refine the derivation of $\lambda$, explore further how $\lambda$ and $\Delta u$ depend on $v$, and test these predictions against observations on bent plates.

\section{Theoretical analysis of shear-band spacing}

While following the analysis by Conner et al. [5], we abandon the assumption of isotropic yielding that is usual in such analyses [5-7]. We derive the stress and strain induced by individual SBs and apply a fully self-consistent approach to derive the most probable SB spacing. We focus on the deformation typical of small MG plates under bending (Fig. 1a), showing an array of SBs that have a characteristic angle $\theta$ with respect to the neutral plane of the bent plate. Such an array of SBs, with spacing $\lambda$, is geometrically necessary to accommodate the applied strain. The SBs are analogous to mode II cracks (and indeed their evolution into cracks may be important in analysing the onset of brittle fracture) [5-7].

We define reference axes $(x, y, z)$ as in Fig. 1b. Taking the shear offset on a SB to be $\Delta u$, the net axial extension or contraction from a symmetric pair of SBs is $2 \Delta u \cos \theta$ (Fig. 1b). For most MGs under simple tension, $\theta$ is slightly greater than $45^{\circ}$. For small deformations, the axial strain $\varepsilon_{x}$ can be decomposed into elastic and plastic components, $\varepsilon_{x}^{\mathrm{e}}$ and $\varepsilon_{x}^{\mathrm{p}}$ :

$\varepsilon_{x}=\varepsilon_{x}^{\mathrm{e}}+\varepsilon_{x}^{\mathrm{p}}$

The distribution of axial stress $\sigma_{x}$ through the thickness of the bent plate has the same form as $\varepsilon_{x}^{\mathrm{e}}$ (Fig. 1c).
Assuming that the strain field around a SB is the same as for a mode II crack, the offset at a SB in a bent plate of half-thickness $h$ is given by:

$\Delta u=\left\{\begin{array}{l}\frac{(1-2 v)}{(1-v) \sin \theta} \frac{a}{R} \sqrt{a^{2}-(y-h)^{2}} \quad \text { if }(h-a)<y \leq h \\ 0 \text { if } 0 \leq y<(h-a),\end{array}\right.$

where $R$ is the bending radius, and $a$ is the projected length of the SB on the $y$-axis (Fig. 1c). Please see the Appendix for the derivation of Eq. (2), which starts from the analysis by Conner et al. [5]. Taking the geometry in Fig. 1b, we can express the plastic strain associated with each shear band as

$\varepsilon_{x}^{\mathrm{p}}=\frac{\Delta u \cos \theta}{(\lambda / \cos \theta)}=\frac{\Delta u \cos ^{2} \theta}{\lambda}$

We limit our consideration to the case where the plate has a large width (i.e. parallel to the $z$ axis); in that case, the bending is in plane strain with $\varepsilon_{z}=0$ (and $\sigma_{y}=0$ ). The axial stress is then given by

$\sigma_{x}=\frac{E \varepsilon_{x}^{\mathrm{e}}}{1-v^{2}}$

where $E$ is Young's modulus. The local strain-energy density (energy per unit volume) $w$ in the bent plate is

$w=\frac{\left(1-v^{2}\right)}{2 E} \sigma_{x}^{2}=\frac{E}{2\left(1-v^{2}\right)}\left(\varepsilon_{x}^{\mathrm{e}}\right)^{2}$

The total elastic strain energy $W$ of the plate is then given by

$W=L B \int_{0}^{h} w d y=\frac{L B E}{\left(1-v^{2}\right)}\left[\int_{0}^{h-a}\left(\frac{y}{R}\right)^{2} d y+\int_{h-a}^{h}\left(\frac{y}{R}-\frac{\Delta u \cos ^{2} \theta}{\lambda}\right)^{2} d y\right]$,

where $L$ is the length of the plate (parallel to $x$ ) and $B$ is its width (parallel to $z$ ). The second integral on the right-hand side of b

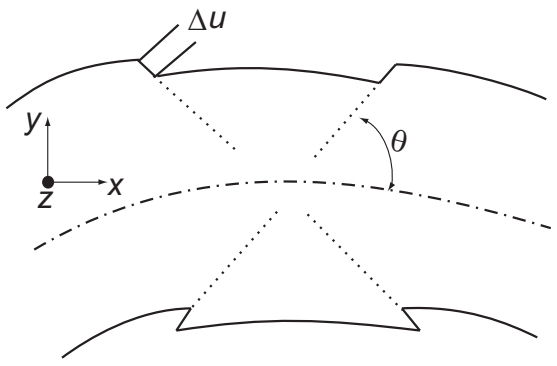

C

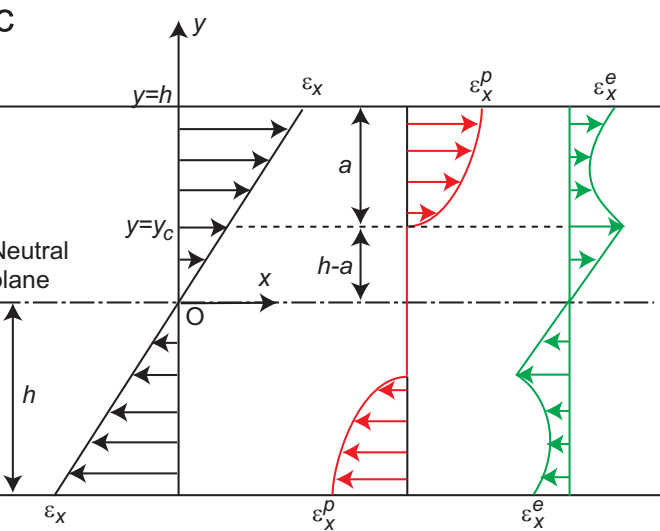

a

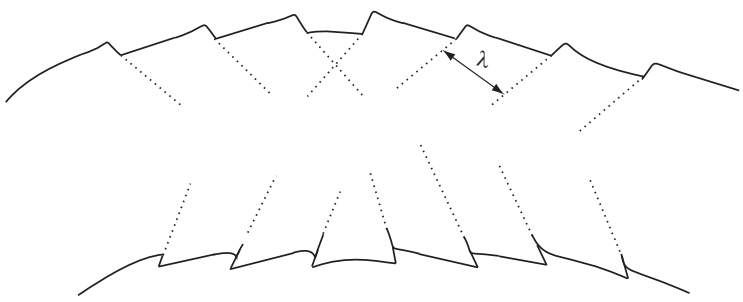

d

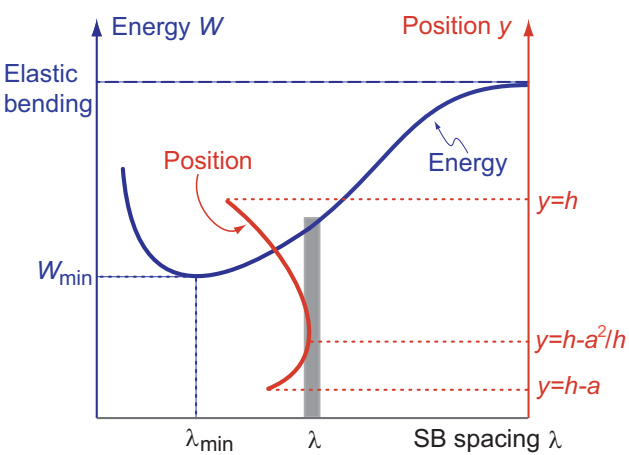

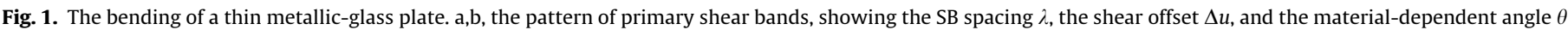

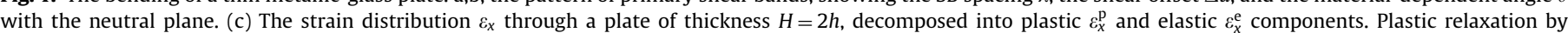

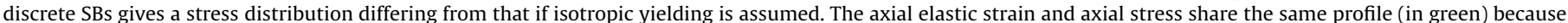

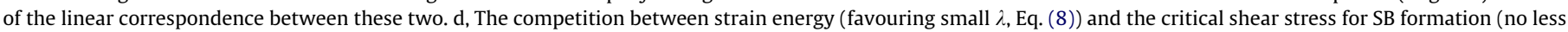

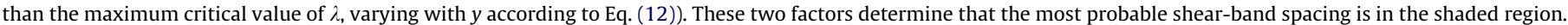
(For interpretation of the references to color in this figure legend, the reader is referred to the web version of this article.) 
Eq. (6), takes account of the plastic strain in the presence of the SB. SBs cease to propagate as they approach the neutral plane. With Eq. (4), noting that $E=2(1+v) \mu$ in an isotropic medium and that $\sigma_{\mathrm{c}}=2 \tau_{\mathrm{c}}$, the transition between the elastic and plastic zones, at the characteristic depth $a$ (Fig. 1c), is determined by calculating the distance from the neutral plane at which the shear stress reaches the critical value for yield $\tau_{\mathrm{c}}$ :

$y_{\mathrm{c}}=R \frac{(1-v) \tau_{\mathrm{c}}}{\mu}$

where $\mu$ is the shear modulus. We have

$a=h-y_{\mathrm{c}}=h-R \frac{(1-v) \tau_{\mathrm{c}}}{\mu}$

The total strain energy in the bent plate then is:

$W=\frac{E B L}{3\left(1-v^{2}\right)}\left[\frac{h^{3}}{R^{2}}-\frac{2 \alpha}{\lambda R}\left(\frac{3 \pi h a^{2}}{4}-a^{3}\right)+\frac{2 \alpha^{2} a^{3}}{\lambda^{2}}\right]$ with

Table 1

The mechanical properties of several metallic glasses with different Poisson's ratio $v$.

\begin{tabular}{lllllll}
\hline Material & $v$ & $\mu(\mathrm{GPa})$ & $\sigma_{c}(\mathrm{GPa})$ & $\tau_{c}(\mathrm{GPa})$ & $\theta$ & $c$ \\
\hline $\mathrm{Zr}_{41} \mathrm{Ti}_{14} \mathrm{Cu}_{12.5} \mathrm{Ni}_{10} \mathrm{Be}_{22.5}[5,14]$ & 0.341 & 36.6 & 1.9 & 0.95 & 52 & 0.034 \\
$\mathrm{Zr}_{57} \mathrm{Nb}_{5} \mathrm{Al}_{10} \mathrm{Cu}_{15.4} \mathrm{Ni}_{12.6}[6,14]$ & 0.362 & 30.1 & 1.8 & 0.9 & $45^{\circ}$ & 0.038 \\
$\left(\mathrm{Cu}_{50} \mathrm{Zr}_{50}\right)_{95} \mathrm{Al}_{5}[24,27]$ & 0.372 & 32.3 & 2.0 & 1.0 & $50^{\circ}$ & 0.039 \\
$\mathrm{Pt}_{57.5} \mathrm{Cu}_{14.7} \mathrm{Ni}_{5.3} \mathrm{P}_{12.5}[15]$ & 0.42 & 33.4 & 1.4 & 0.7 & 50 & 0.024 \\
$\mathrm{Pt}_{74.7} \mathrm{Cu}_{1.5} \mathrm{Ag}_{0.3} \mathrm{P}_{18} \mathrm{~B}_{4} \mathrm{Si}_{1.5}[16]$ & 0.43 & 32.4 & 1.2 & 0.6 & 50 & 0.021
\end{tabular}
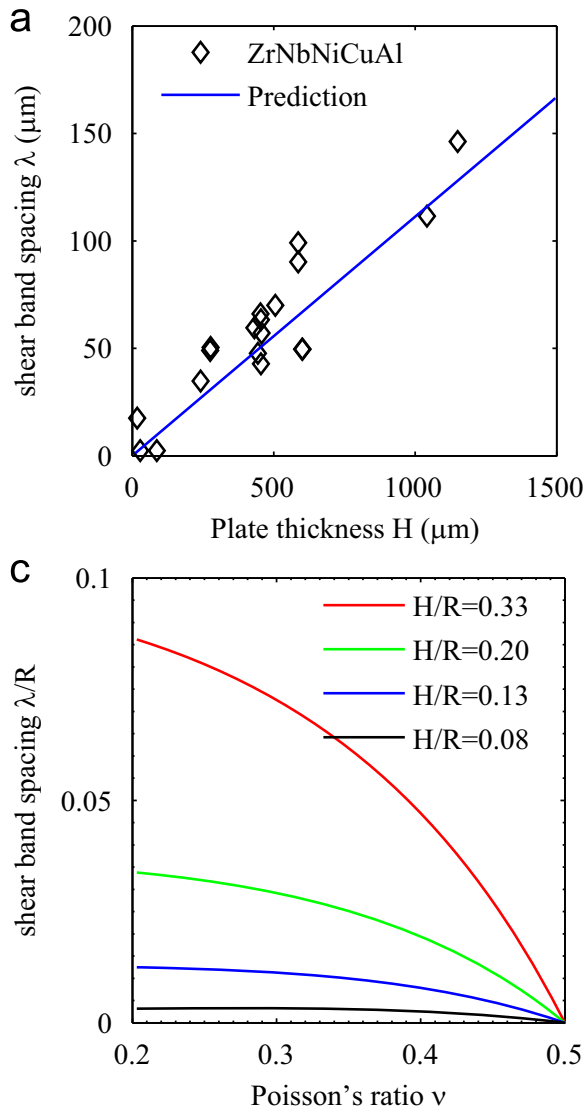

$\alpha=\left(\frac{1-2 v}{1-v}\right) \frac{a}{R} \frac{\cos ^{2} \theta}{\sin \theta}$

Plastic flow mediated by SBs lowers the elastic strain energy, and it is expected that the smaller the SB spacing, the more complete is the strain relaxation and the lower the total energy. Interestingly, Eq. (9) shows that $W$ does not decrease monotonically as $\lambda$ decreases, instead showing a minimum at

$\lambda_{\min }=\frac{8 \alpha R a}{3 \pi h-4 a}$

The increase in $W$ below $\lambda_{\min }$ is due to the unconstrained elastic strain in our derivation. To enable a SB to extend from the surface to a depth $a$, the axial elastic strain should never be negative; this requires that in the range $(h-a) \leq y \leq h$,

$\varepsilon_{x}^{\mathrm{e}}=\frac{y}{R}-\frac{\Delta u \cos ^{2} \theta}{\lambda} \geq 0$,

from which we have

$\lambda \geq \frac{\cos ^{2} \theta}{\sin \theta}\left(\frac{1-2 v}{1-v}\right) \frac{a}{y} \sqrt{a^{2}-(y-h)^{2}}$

The maximum value on the right-hand side in Eq. (12) occurs at $y_{\max }=h-\left(a^{2} / h\right)$, satisfying $(h-a) \leq y \leq h$. This maximum at $y_{\max }$ represents the minimum possible $\lambda$ if negative $\varepsilon_{x}^{\mathrm{e}}$ is to be avoided throughout the plate thickness. As illustrated in Fig. 1d, this condition determines the most probable SB spacing, which is:

$\lambda=\frac{\cos ^{2} \theta}{\sin \theta}\left(\frac{1-2 v}{1-v}\right) \frac{a^{2}}{\sqrt{h^{2}-a^{2}}}$
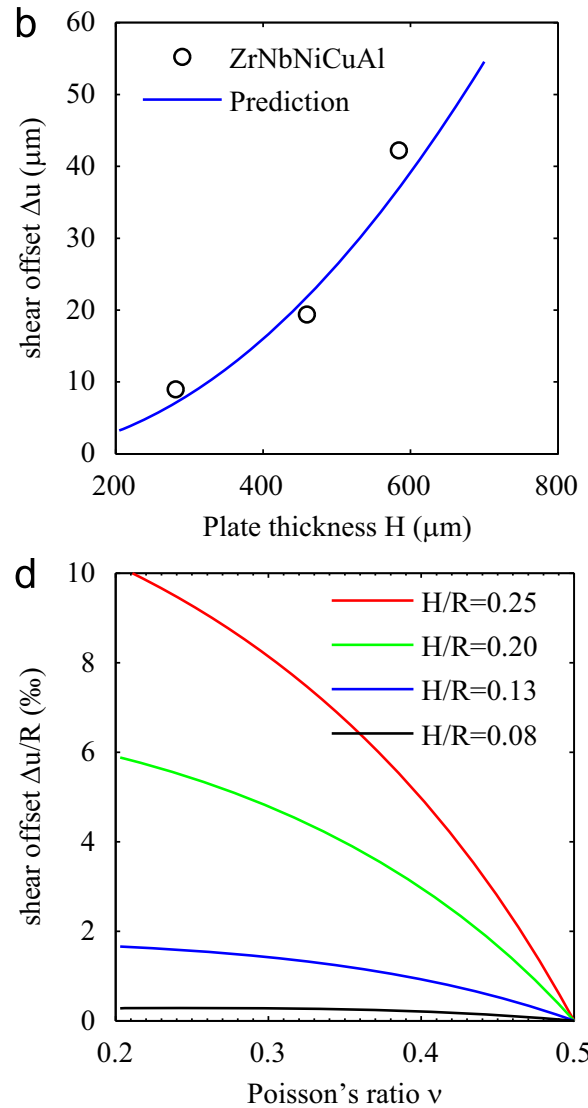

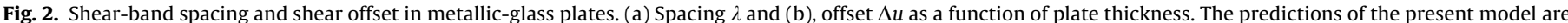

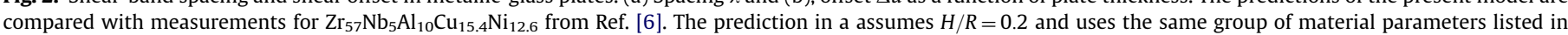

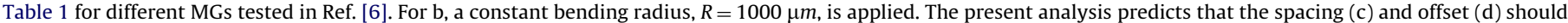
depend on Poisson's ratio $v$, each tending to zero as $v \rightarrow 0.5$. 
This is different from the expression for $\lambda$ derived by Conner et al. [5]. Using Eq. (8), and taking plate thickness $H=2 h$, we can rewrite Eq. (13) as

$\frac{\lambda}{R}=\beta \frac{(1-2 v)}{(1-v)} \frac{((H / R)-c)^{2}}{\sqrt{(2 c H / R)-c^{2}}} \quad$ with $\quad c=(1-v) \frac{\sigma_{\mathrm{c}}}{\mu}$,

where $\beta=\cos ^{2} \theta / 2 \sin \theta$ is a material-dependent coefficient. It is clear that $\lambda / R$ is controlled by four dimensionless parameters: $H / R$, $\sigma_{c} / \mu, \theta$ and $v$. For small $c, \lambda / R$ is proportional to $(H / R)^{3 / 2}$. The shear offset $\Delta u$ is given by

$\frac{\Delta u}{R}=\gamma\left(\frac{1-2 v}{1-v}\right)\left(\frac{H}{R}-c\right)^{2}$,

where $\gamma=1 /(4 \sin \theta)$. For small $c, \Delta u / R$ is proportional to $(H / R)^{2}$, consistent with the work of Conner et al. [5]. Johnson and Samwer [23] found that at room temperature $\sigma_{c} / \mu$ has the same value for most metallic glasses. From Eqs. (14) and (15), we see that both SB spacing and shear offset depend only on $R, H / R, \theta$ and $v$. Next, we use Eqs. (14) and (15) to analyze data from the literature [6] and from experiments in the present work. Poisson's ratio and other materials properties of the metallic glasses under consideration are given in Table 1.
In Fig. 2a, we show our prediction of $\lambda$ for MG plates of different thicknesses under bending. Measurements by Conner et al. [6] on bent plates of $\mathrm{Zr}_{57} \mathrm{Nb}_{5} \mathrm{Al}_{10} \mathrm{Cu}_{15.4} \mathrm{Ni}_{12.6}$ are shown. Without any fitting parameter, Eq. (14) matches the measured dependence of spacing on plate thickness. We have assumed that $H / R, \tau_{c} / \mu, v$ and $\theta$ have uniform values across all the tests. Fig. $2 b$ shows the prediction for the dependence of $\Delta u$ on plate thickness at a constant bending radius of $R=1000 \mu \mathrm{m}$. For fixed $R$, Eq. (15) predicts a parabolic increase of offset with increasing plate thickness, in good agreement with experiment from [6].

Eqs. (14) and (15) show that, other factors being constant, both $\lambda$ and $\Delta u$ are proportional to $(1-2 v) /(1-v)$. Conner et al. [5] obtained this result but did not explore it, as their work preceded the current intense interest in how the plasticity of MGs is correlated with their elastic properties (particularly $v$ ) [14-16, 24-26]. We now calculate the dependence of $\lambda$ (Fig. 2c) and of $\Delta u$ (Fig. 2d) on $v$ for several bending ratios. All other materials parameters are assumed to be the same as those of $\mathrm{Zr}_{41} \mathrm{Ti}_{14} \mathrm{Cu}_{12.5}$ $\mathrm{Ni}_{10} \mathrm{Be}_{22.5}$ (in Table 1). The calculations imply that in metallic glasses with higher $v$, plasticity is accommodated by denser arrays of SBs with smaller shear offsets, i.e. the deformation is more uniform.

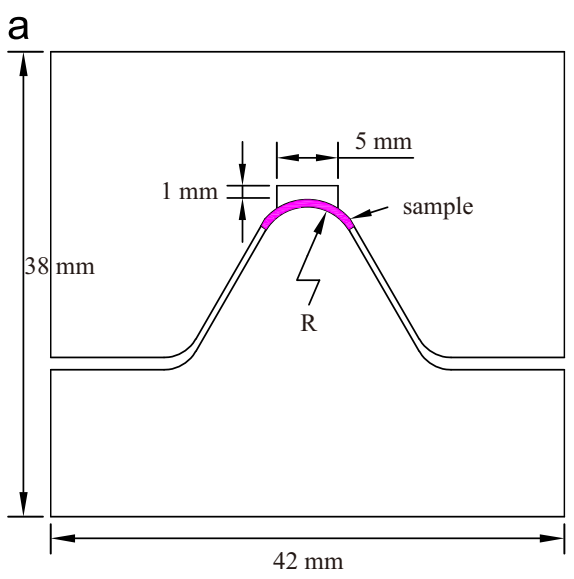

C

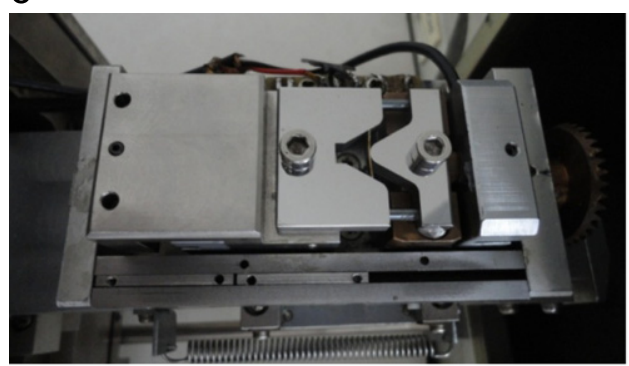

e

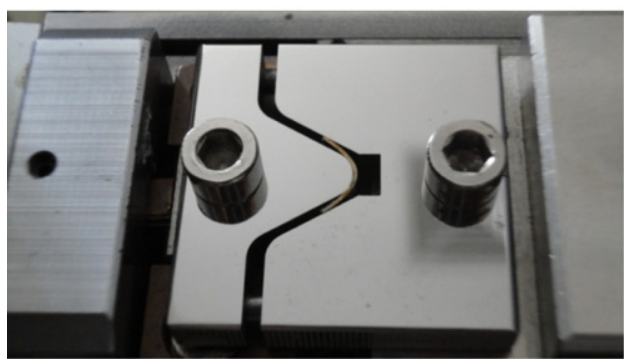

b

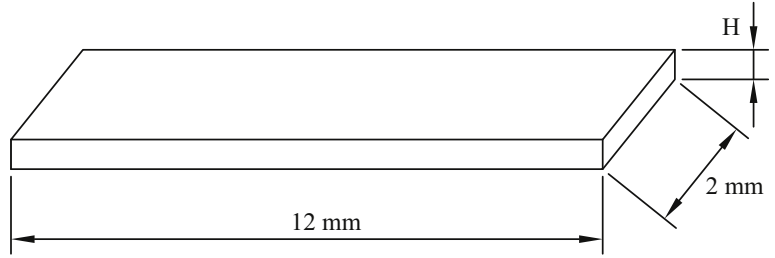

d

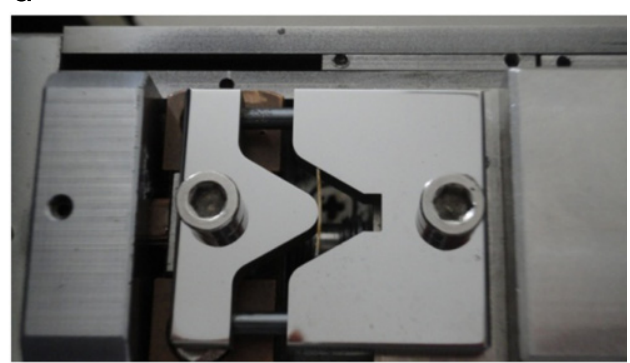

Fig. 3. Experimental setup for in-situ bending test. (a) ben
(d) close-up of the testing region and (e) a bent sample. 
a

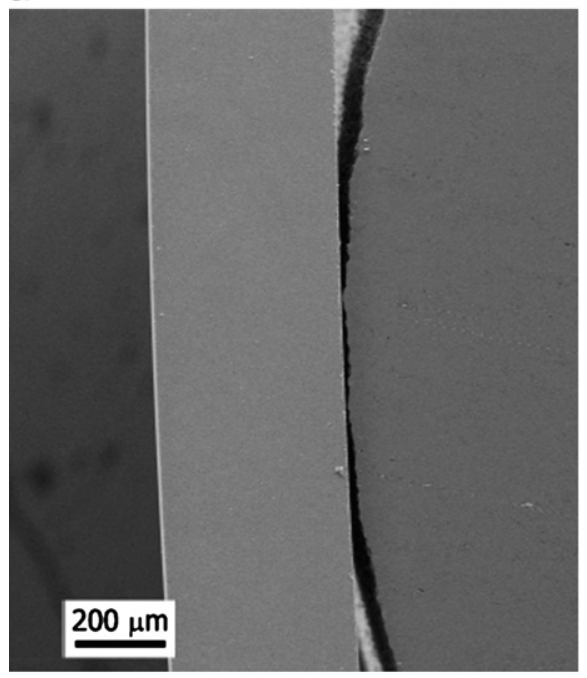

C

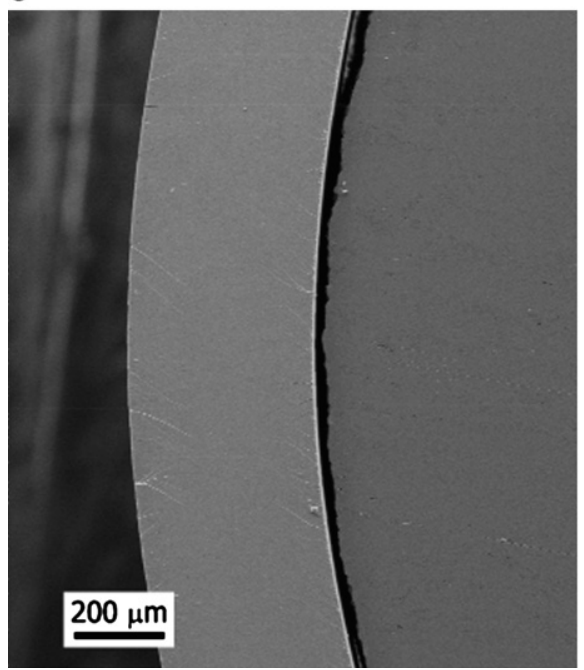

b

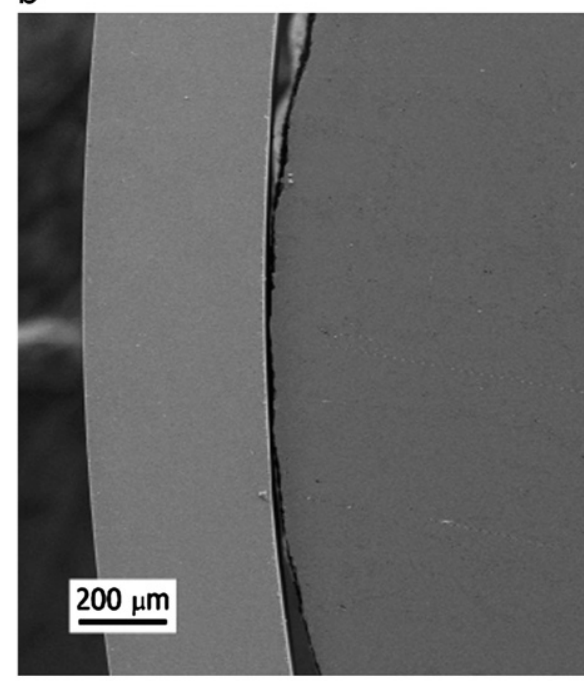

d

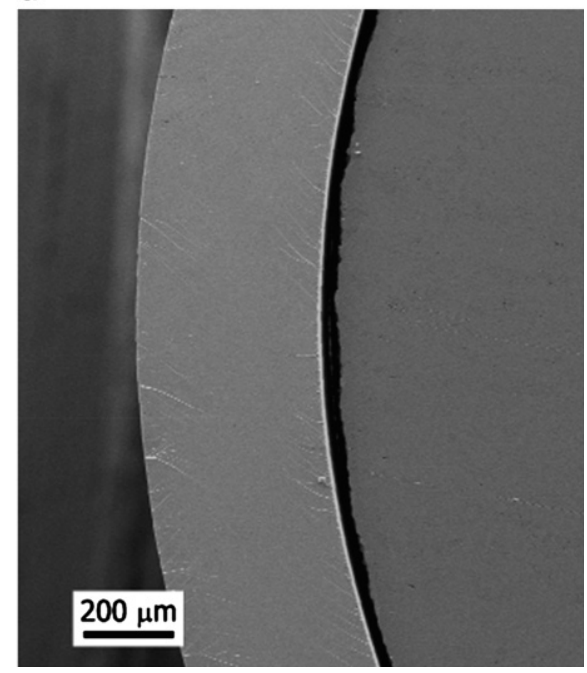

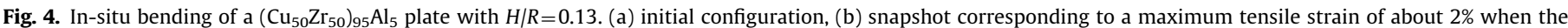

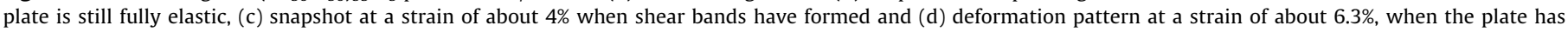
conformed to the curved loading tip.

\section{Experimental methods}

The link between the plasticity of MGs and their Poisson's ratio is further explored in in-situ bending experiments. Two types of metallic glass, $\mathrm{Zr}_{41} \mathrm{Ti}_{14} \mathrm{Cu}_{12.5} \mathrm{Ni}_{10} \mathrm{Be}_{22.5}$ (Vitreloy 1) and $\left(\mathrm{Cu}_{50} \mathrm{Zr}_{50}\right)_{95} \mathrm{Al}_{5}$ are tested in the present work. Both MGs are made in an arc-melter with a water-cooled hearth under a titanium-gettered argon atmosphere. Elemental metals ( $>99.9 \%$ purity) are used to form the master alloy, and suction-cast into $1 \mathrm{~mm} \times 10 \mathrm{~mm} \times 60 \mathrm{~mm}$ plates. To ensure good curvature control in the bent region, we designed the bending rig shown Fig. 3a.

Typical sample dimensions are noted in Fig. 3b; different plate thicknesses were tested, ranging from $0.25 \mathrm{~mm}$ to $1 \mathrm{~mm}$. The samples are cut from as-cast metallic glasses using electrical discharge machining (EDM). To remove possible surface damage during machining, the original samples were intentionally about $0.2 \mathrm{~mm}$ thicker than the target thickness, and then mechanically ground using 5000 grade sandpaper on four surfaces (all except the ends of the plate), followed by electropolishing. We use five percent (vol\%) perchloric acid ethanol solution as the electrolyte, $29 \mathrm{~V}$ and $0.3 \mathrm{~A}$ as the electrical parameters; samples are clamped from the two ends by stainless tweezers as the anode, and then electro-polished for about $15 \mathrm{~s}$ at $250 \mathrm{~K}$. After polishing, samples are processed by ultrasonic cleaning in ethanol solutions immediately. After sample preparation, the samples were tested in an in-situ bending rig that we designed. The magnitude of the maximum strain rate, which occurs near the out-most surfaces of bending plates, is about $10^{-3} / \mathrm{s}$. The set-up of this rig in a Hitachi S-570 Scanning Electron Microscope (SEM) is shown in Fig. $3 c-e$, which respectively show the constraint of the sample and the sample after bending. Fig. 4 shows the gradual bending of a $\left(\mathrm{Cu}_{50} \mathrm{Zr}_{50}\right)_{95} \mathrm{Al}_{5}$ plate with $H / R=0.13$. Four stages: initial bending, at the elastic limit, nucleation of shear bands, and the final bent plate, are shown in Figs. $4 a-d$.

After obtaining the bent plate at a given bending ratio, we measured about 20 independent shear-band spacings or shear offsets. The average of the measurements is used for most probable shear-band spacing and for shear offset.

\section{Application of the theory}

Shear bands in Vitreloy 1 [2] bent to $H / R=0.1$ and in $\left(\mathrm{Cu}_{50} \mathrm{Zr}_{50}\right)_{95}$ $\mathrm{Al}_{5}$ bent to $H / R=0.16$, are shown in Figs. $5 \mathrm{a}$ and b, respectively. 
a

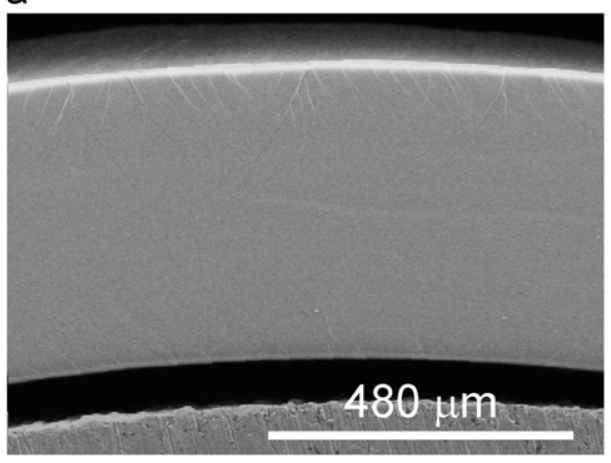

C

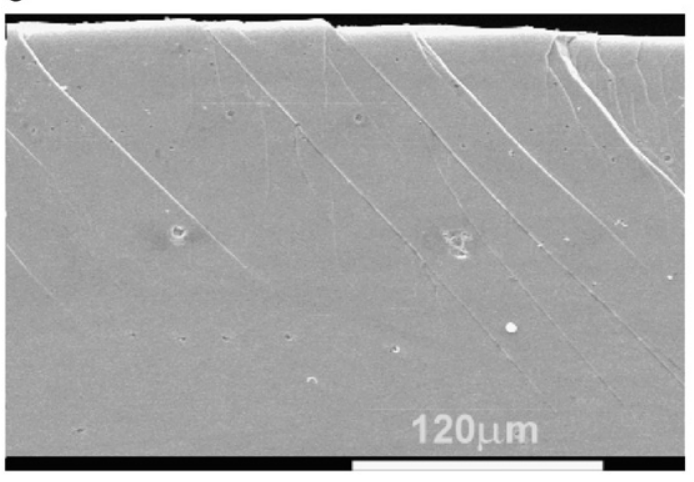

b

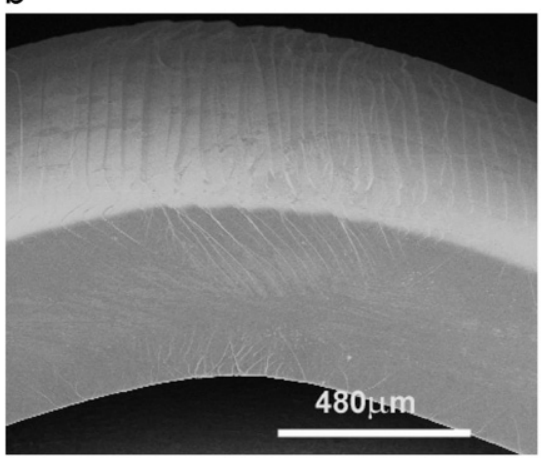

d

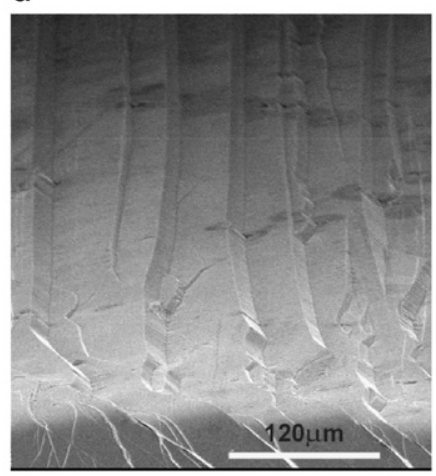

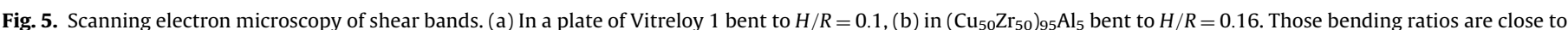

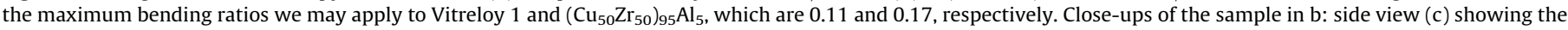
spacing of primary shear bands, top view (d) showing the shear offsets which, though large, do not indicate any incipient cracking.
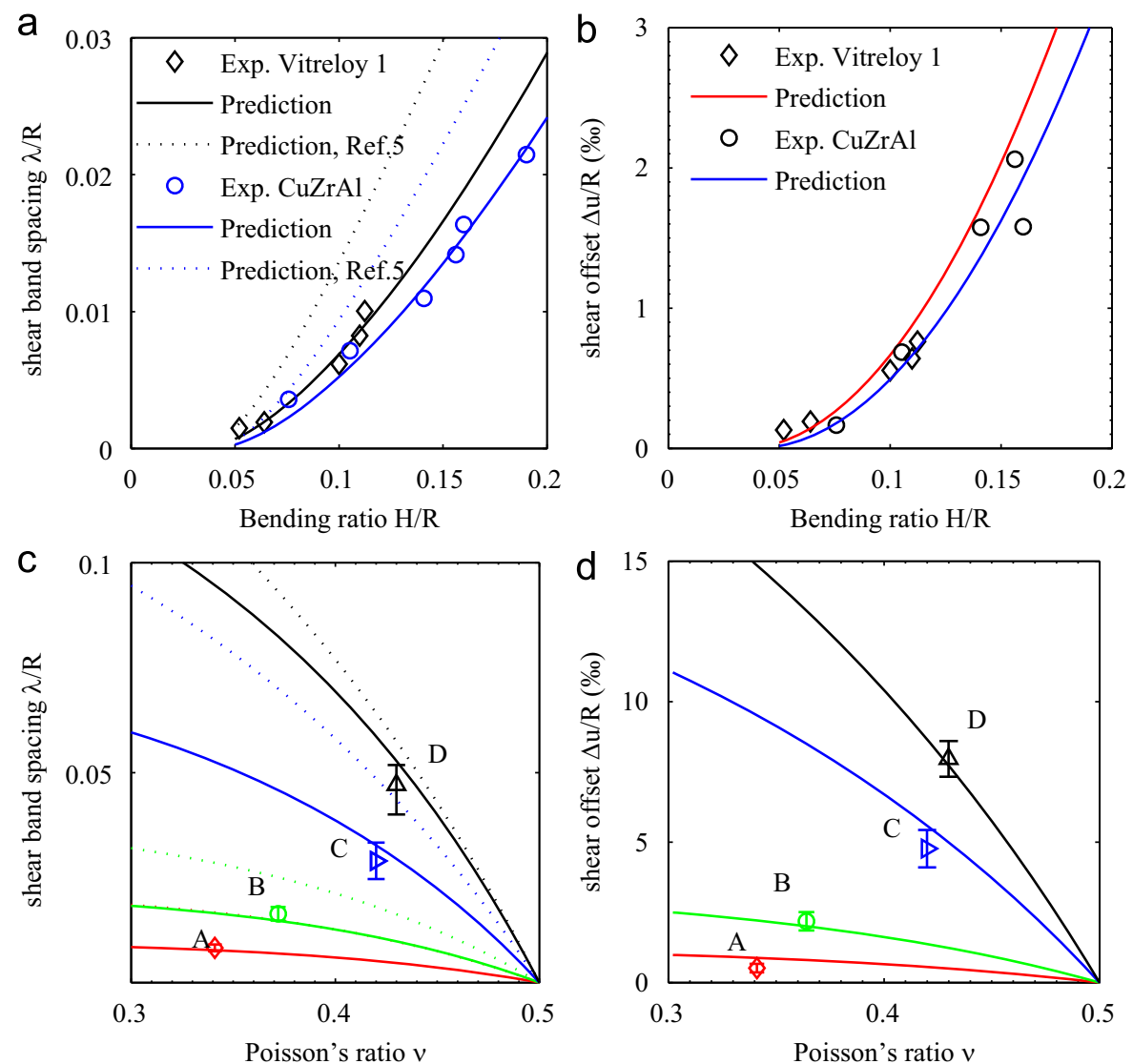

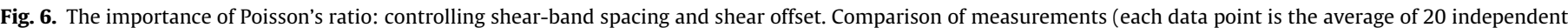

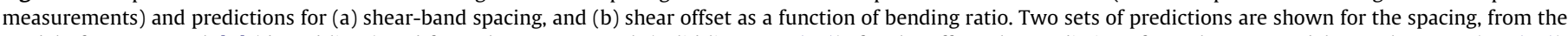

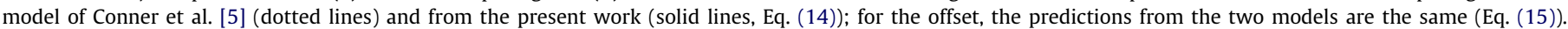

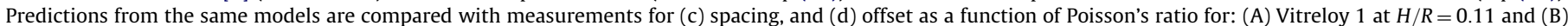

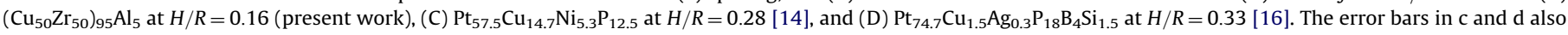
represent the standard deviation of 20 measurements. 
For the latter case, close-up views of the side (Fig. 5c) and top (d) of the bent plate clearly show the SBs, their spacing and the associated offsets. Fig. 6 shows the matching between these experimental results and our predictions for $\lambda$ (Fig. 6a), and $\Delta u$ (Fig. 6b) as a function of bending ratio. For four metallic glasses with distinct Poisson's ratios (materials parameters in Table 1), our predictions for $\lambda$ (Fig. 6c) and $\Delta u$ (Fig. 6d) as a function of $v$ at different bending ratios are in good agreement with experiment. For $\lambda$, the predictions in the present work differ from those of Conner et al. [5]. From Figs. 6a and 6c, it appears that the predictions of Conner et al., while capturing the trend of the data, systematically overestimate the values of $\lambda$. This may be because their analysis ignores the contribution of elastic strain (Eq. (1)) that is relatively much more significant in MGs than in polycrystalline metals [23].

\section{Concluding remarks}

The pioneering work of Conner et al. [5] has been adapted and extended in the present work to permit quantitative comparison of prediction with measurement of shear-band spacings in bent plates of metallic glasses with a range of values of Poisson's ratio $v$. Higher $v$ is found to make the plastic deformation of metallic glasses more uniform. This is desirable in itself, and the resulting smaller shear offsets are particularly significant in giving shear bands that are more likely to operate cold (without significant heating) [28], with greater energy absorption (correlated with toughness), and less likely to develop into cracks [5]. Such effects extend plasticity before failure, intriguingly the same effect of higher $v$ as for polycrystalline metals [12], even though the flow mechanisms in the two classes of material are entirely distinct (dislocation glide in polycrystals, shear banding in glasses). The predictions suggest that plastic flow in metallic glasses would become completely uniform (implying a suppression of shear banding) when $v$ rises to its limit of 0.5 (the value for a liquid). In isotropic glasses the highest $v$ obtained so far [16] is 0.43 , and induced anisotropy may permit even higher values to be reached in particular directions [29]. The present work points towards quite exceptional mechanical properties of metallic glasses as $v$ is increased towards the limit

\section{Acknowledgements}

The authors acknowledge support from the Chinese Academy of Sciences, NSF of China (11021262), and MOST 973 of China

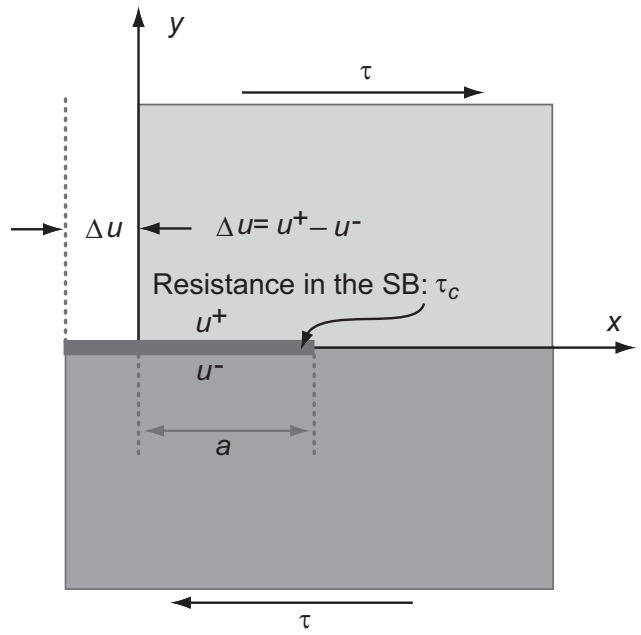

Fig. A1. The displacement discontinuity across a shear band with constant shear resistance $\tau_{c}$
(Nr. 2012CB937500) for YW, from MOST 973 of China (Nr. 2010CB731603) and the NSF of China (50921091) for WHW, and from the EPSRC for ALG.

\section{Appendix. The offset on a shear band}

Our expressions for the shear offset $\Delta u$ are entirely consistent with those derived by Conner et al. [5]. The derivation of Eq. (2) in the main text is based on Eq. (15) in Ref. [5].

For a constant shear resistance $\tau_{c}$ (Fig. A1), the applied shear stress induces a displacement jump across the shear-band plane, given by:

$\Delta u=\left\{\begin{array}{l}\frac{(1-2 v)}{\mu}\left(\tau-\tau_{c}\right) \sqrt{a^{2}-x^{\prime 2}} \text { if } x^{\prime} \leq a \\ 0 \text { if } x^{\prime}>a .\end{array}\right.$

Eq. (15) in Ref. [5] is obtained based on the configuration shown in Fig. A1 (the shear-band length is $a$, and $x^{\prime}$ points to its leading edge). If we convert Eq. (15) in Ref. [5] to our inclined shear bands with a coordinate system defined in Fig. 1b,c, we have

$\Delta u=\left\{\begin{array}{cl}\frac{(1-2 v)}{\mu}\left(\tau-\tau_{c}\right) \frac{\sqrt{a^{2}-(y-h)^{2}}}{\sin \theta} & \text { if }(h-a)<y \leq h \\ 0 & \text { if } 0 \leq y<(h-a),\end{array}\right.$

which can be further rewritten as:

$\Delta u=\left\{\begin{array}{ccc}\frac{(1-2 v)}{\mu}\left(\frac{\sigma(y=h)}{2}-\frac{\sigma_{c}}{2}\right) \frac{\sqrt{a^{2}-(y-h)^{2}}}{\sin \theta} & \text { if } \quad(h-a)<y \leq h \\ 0 & \text { if } \quad 0 \leq y<(h-a),\end{array}\right.$

if we use the same approximation adopted in Eqs. (16) and (17) of Ref. [5]. For Eq. (A3), recall that we have

$\varepsilon_{x}=\frac{1-v}{2 \mu} \sigma_{x}$

This is the same as Eq. (2) in Ref. [5], and Eq. (A3) can then be expressed as

$\Delta u=\left\{\begin{array}{cll}\frac{(1-2 v)}{(1-v)}\left(\varepsilon_{x}(y=h)-\varepsilon_{c}\right) \frac{\sqrt{a^{2}-(y-h)^{2}}}{\sin \theta} & \text { if } & (h-a)<y \leq h \\ 0 & \text { if } & 0 \leq y<(h-a)\end{array}\right.$

With Eq. (A5), we arrive at Eq. (2) by using $\varepsilon_{x}(y=h)=h / R$ and the critical yield strain $\varepsilon_{c}=(h-a) / R$.

$\Delta u=\left\{\begin{array}{ccc}\frac{(1-2 v)}{(1-v) \sin \theta} \frac{a}{R} \sqrt{a^{2}-(y-h)^{2}} & \text { if } & (h-a)<y \leq h \\ 0 & \text { if } & 0 \leq y<(h-a)\end{array}\right.$

\section{References}

[1] A.L. Greer, Science 267 (1995) 1947-1953.

[2] W.L. Johnson, MRS Bull. 24 (1999) 42-56.

[3] A. Inoue, Acta Mater. 48 (2000) 279-306.

[4] C.A. Schuh, T.C. Hufnagel, U. Ramamurty, Acta Mater. 55 (2007) 4067-4109

[5] R.D. Conner, W.L. Johnson, N.E. Paton, W.D. Nix, J. Appl. Phys. 94 (2003) 904-911.

[6] R.D. Conner, Y. Li, W.D. Nix, W.L. Johnson, Acta Mater. 52 (2004) 2429-2434.

[7] G. Ravichandran, A. Molinari, Acta Mater. 53 (2005) 4087-4095.

[8] J.X. Zhao, F.F. Wu, Z.F. Zhang, Mater. Sci. Eng. A 527 (2010) 6224-6229.

[9] T. Wada, A. Inoue, Mater. Trans. 45 (2004) 2761-2765.

[10] A.H. Brothers, D.C. Dunand, Adv. Mater. 17 (2005) 484-486.

[11] H.-J. Kim, S. Grossi, Y.G. Kweon, Wear 232 (1999) 51-60.

[12] S.F. Pugh, Philos. Mag. 45 (1954) 823-843.

[13] H.S. Chen, J.T. Krause, E. Coleman, J. Non-Cryst. Solids 18 (1975) 157-171.

[14] J.J. Lewandowski, W.H. Wang, A.L. Greer, Phil. Mag. Lett. 85 (2005) 77-87.

[15] J. Schroers, W.L. Johnson, Phys. Rev. Lett. 93 (2004) 255506

[16] M.D. Demetriou, M. Floyd, C. Crewdson, J.P. Schramm, G. Garrett W.L. Johnson, Scripta Mater. 65 (2011) 799-802.

[17] M.D. Demetriou, M.E. Launey, G. Garrett, J.P. Schramm, D.C. Hofmann W.L. Johnson, R.O. Ritchie, Nat. Mater. 10 (2011) 123-128.

[18] A. Kelly, W.R. Tyson, A.H. Cottrell, Philos. Mag. 15 (1967) 567-586. 
[19] J.R. Rice, R. Thomson, Philos. Mag. 29 (1974) 73-97.

[20] Z.W. Shan, J. Li, Y.Q. Cheng, A.M. Minor, S.A. Syed Asif, O.L. Warren, E. Ma, Phys. Rev. B 77 (2008) 155419.

[21] C.A. Volkert, A. Donohue, F. Spaepen, J. Appl. Phys. 103 (2008) 083539.

[22] D. Jang, J.R. Greer, Nat. Mater. 9 (2010) 215-219.

[23] W.L. Johnson, K.A. Samwer, Phys. Rev. Lett. 95 (2005) 195501.

[24] W.H. Wang, J. Appl. Phys. 99 (2006) 093506.
[25] A.L. Greer, Nat. Mater. 10 (2011) 88-89.

[26] G.N. Greaves, A.L. Greer, R.S. Lakes, T. Rouxel, Nat. Mater. 10 (2011) 823-837.

[27] P. Yu, H.Y. Bai, Mater. Sci. Eng. A 485 (2008) 1-4.

[28] Y.Q. Cheng, Z. Han, Y. Li, E. Ma, Phys. Rev. B 80 (2009) 134115.

[29] A. Concustell, S. Godard-Desmarest, M.A. Carpenter, N. Nishiyama, A.L. Greer, Scripta Mater. 64 (2011) 1091-1094. 